\title{
Host genetics of coronavirus infection
}

\section{c6 \\ these studies \\ provide valu- \\ able leads for \\ therapeutic investigation}

Characterizing the role of host genetics in SARS coronavirus 2 (SARS-CoV-2) infection may help to understand the diverse clinical outcomes of infected individuals, as well as providing mechanistic information on host-virus interactions and potential drug targets. Two new reports in Cell use genomewide CRISPR screens to uncover host determinants of coronavirus infection, identifying potential leads for antiviral therapeutics.

In their study, Daniloski, Jordan et al. used human A549 alveolar lung cancer cells ectopically overexpressing the SARS-CoV-2 entry receptor, ACE2. They infected these cells with a pooled genome-wide CRISPR library, with each construct encoding a Cas9 nuclease and a guide RNA (gRNA) to direct the disruption of each human target gene. The authors then infected the CRISPR-perturbed cells with SARS-CoV-2 and identified gRNA constructs that were enriched; the targets of these gRNAs are potential infectivity-enhancing genes, the knockout of which protects from cytotoxic SARS-CoV-2 infection.

As proof of principle of the screen's performance, known determinants of SARS-CoV-2 infection were identified, such as the entry receptor ACE2. For a wider view, gene set enrichment analysis revealed several

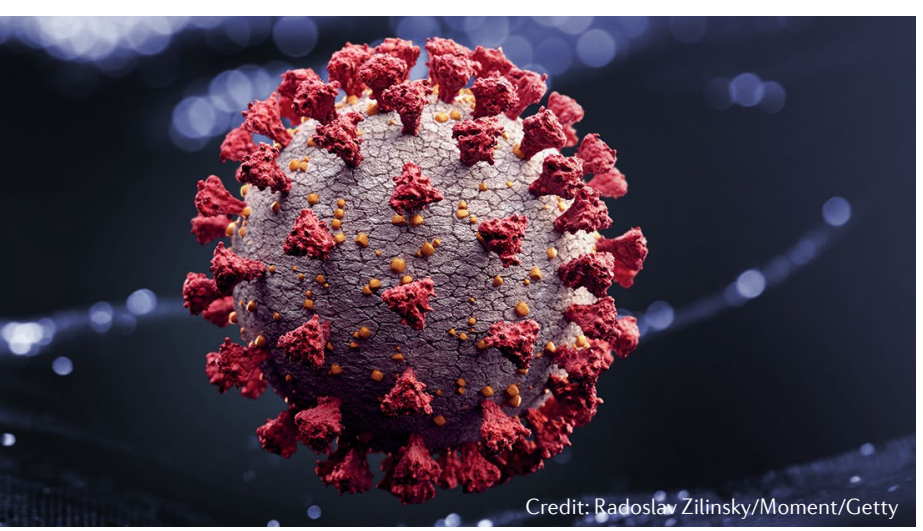

functional groups of hits, particularly those involved in endocytosis and endosomal trafficking, which may be part of the intracellular life cycle of SARS-CoV-2. Indeed, several of the hits were known to have direct protein-protein interactions with SARS-CoV-2 proteins from a previously published proteomics analysis. Additionally, some of the antiviral effects of trafficking-related hits may be through reduced cell-surface delivery of ACE2, which the authors demonstrated for knockout of the RAB7A GTPase.

As loss of function of the gene hits is antiviral, small-molecule inhibition of these targets could be a valuable therapeutic strategy. The team demonstrated antiviral effects of seven inhibitors targeting screen hits, including four inhibitors of PIK3C3.

For insights into the molecular rewiring underlying the antiviral effects, the authors showed using single-cell RNA sequencing that antiviral CRISPR perturbations commonly resulted in upregulation of cholesterol biosynthesis genes. Further to the seven identified inhibitors, the cholesterol-enhancing drug amlodopine also had antiviral effects.

In a separate study, Wei et al. used genome-wide CRISPR screening but sought to understand host susceptibility across all the major recent human outbreak coronaviruses: SARS-CoV, MERS-CoV and SARS-CoV-2.

The authors chose a cell line from Chlorocebus sabaeus monkeys that has endogenous expression of ACE2 (the receptor for both SARS-CoV and SARS-CoV-2) and DPP4 (the receptor for MERS-CoV). CRISPR reagents were delivered in two steps: first Cas9 and then a genome-wide library of C. sabaeus-targeted gRNAs. Following infection with each single coronavirus, cells were analysed for the most enriched and depleted gRNAs.
Hits from the screens were commonly connected to viral entry. For example, ACE2 was the strongest resistance-conferring knockout for the screens involving SARS-CoV-2 and a SARS-CoV-related virus, whereas DPP4 was the top hit for the screen with MERS-CoV. Moreover, a screen with vesicular stomatitis virus, engineered to encode the SARS-CoV-2 spike protein, had substantial overlap with hits from the SARS-CoV-2 screen, despite these viruses only sharing an entry-related protein.

Analysis of the functions of the SARS-CoV-2 gene hits revealed diverse roles in chromatin regulation, such as histone modifiers and chromatin remodeller subunits. One potential antiviral mechanism is through transcriptional downregulation of ACE2, such as for HMGB1 knockout, which led to reduced chromatin accessibility at the ACE2 locus and lower ACE2 expression.

For therapeutic relevance, Wei et al. showed SARS-CoV-2 antiviral effects of three small molecules targeting their screen hits.

Considering the studies together, although both teams validated several of their hits in alternative cell lines, one notable feature is the limited overlap of hits between the studies. Additional CRISPR screens, such as those currently available as preprints, will help to delineate any common versus system-specific effects.

Overall, these studies provide valuable leads for therapeutic investigation. Given the importance of the immune system for both antiviral defence and fatal COVID pathology, it will be interesting to see whether future screens can incorporate immune-system components for a more complete understanding of host-virus interactions.

Darren J. Burgess

ORIGINAL ARTICLES Daniloski, Z. et al. Identification of required host factors for SARS-CoV-2 infection in human cells. Cell https:// doi.org/10.1016/j.cell.2020.10.030 (2020) | Wei, J. et al. Genome-wide CRISPR screens reveal host factors critical for SARS-CoV-2 infection. Cell https://doi.org/10.1016/j.cell.2020.10.028 (2020) 